Check for updates

Cite this: Phys. Chem. Chem. Phys., 2017, 19, 9669

Received 5th January 2017, Accepted 17th March 2017

DOI: 10.1039/c7cp00092h

rsc.li/pccp

\section{The aromaticity of dicupra[10]annulenes}

\author{
Rafael Grande-Aztatzi, (D)*a Jose M. Mercero, ${ }^{\text {b }}$ Eduard Matito, (D) ac \\ Gernot Frenking ${ }^{\text {ad }}$ and Jesus M. Ugalde*a
}

\begin{abstract}
An extensive theoretical investigation of the electronic structure of a tested fair model dicupra[10]annulene compound, based on the analysis of atom-pair delocalization indices, Bader's molecular graph, the inspection of the canonical molecular orbitals, the $z$ components of their Nuclear Independent Chemical Shifts, $\mathrm{NICS}(0)_{z z}$, and the normalized Giambiagi multicenter delocalization indices, concludes that the perimeter aromaticity of the dicupra[10]annulene ring is consistent with both 10 and $14 \pi$-electron Hückel aromatic 10-membered rings. In either case, the 10-membered ring encloses two $6 \pi$-electron aromatic inner rings, hinged at the $\mathrm{Cu}-\mathrm{Cu}$ bond. This work demonstrates that the aromaticity of dicupra[10]annulenes closely resembles that of naphthalene. Hence, they are best regarded as metalla-polyacenes, which could make the building blocks of extended structures such as metalated nanotubes.
\end{abstract}

\section{Introduction}

Wei et al. recently reported the synthesis of dicupra[10]annulenes from the reaction of dilithio reagents with $\mathrm{Cu}(\mathrm{I})$ salts, see Fig. 1, yielding air-sensitive, but thermally stable compounds under inert conditions, which were characterized by X-ray diffraction and X-ray photoelectron spectroscopy. ${ }^{1}$ They found that the 10-membered ring 2 is marginally distorted by the presence of bulky substituents and the four Li atoms, located above and below the annulene ring, and claimed that this species can be best seen as a $10 \pi$-electron Hückel aromatic ring.

Aromaticity in metal containing compounds constitutes a topic of considerable interest, both fundamental ${ }^{2-5}$ and practical., Indeed, the idea of substituting a $\mathrm{C}-\mathrm{H}$ unit of an aromatic hydrocarbon by an isolobal transition metal was first put forward by Thorn and Hoffmann in $1979,{ }^{8}$ and has continued to be inspirational for the synthesis of metal containing aromatic compounds. ${ }^{9}$

The research of Wei et al. is remarkable because it makes one further step ahead, since it turns, formally, a nonaromatic [10]annulene species into an aromatic, metal-containing, dicupra[10]annulene. Recall that [10]annulenes are nonaromatic species in spite of being $10 \pi$-electron systems. It is the steric hindrance of the two internal hydrogens that prevents

\footnotetext{
${ }^{a}$ Kimika Fakultatea, Euskal Herriko Unibertsitatea (UPV/EHU) and Donostia International Physics Center (DIPC), P.K. 1072, 20080 Donostia, Euskadi, Spain. E-mail: aztatzi26@gmail.com, jesus.ugalde@ehu.eus

${ }^{b}$ IZO-SGI SGiker, Kimika Fakultatea, Euskal Herriko Unibertsitatea (UPV/EHU), P.K. 1072, 20080 Donostia, Euskadi, Spain

${ }^{c}$ IKERBASQUE, Basque Foundation for Science, 48011 Bilbao, Euskadi, Spain

${ }^{d}$ Fachberich Chemie, Philipps-Universität Marburg, Hans-Meerwein-Strasse 4, 35043 Marburg, Germany
}

delocalization of the $\pi$ electrons around the ring. Replacement of these $\mathrm{C}-\mathrm{H}$ units by isolobal metals could, in principle, release such hindrance and allow for $\pi$ electron full delocalization around the ring, yielding a $10 \pi$ electron Hückel aromatic system.

In this work we analyze in depth the electronic structure of a model compound of 2 , where the $\mathrm{R}$ and the TMS substituents have been replaced by the computationally more convenient $\mathrm{H}$ atoms. Our aim is to unveil the electron delocalization pattern along the ring fragment.

\section{Methods}

Density functional theory (DFT) has been used to optimize the structure of the dicupra[10]annulene 2 with $\mathrm{R}=\mathrm{H}$ and the TMS substituents replaced by $\mathrm{H}$ atoms, in order to make it computationally more accessible, while retaining the chemical bonding features of its ring moiety. Subsequently, we have calculated the vibrational spectra and inspected that all vibrational modes have positive force constants. This confirms that the calculated structure corresponds to a minimum energy structure, at the level of theory employed.

These calculations have been carried out using the Gaussian09 package ${ }^{10}$ and the hybrid exchange-correlation functional PBE0 ${ }^{11}$ and the def2-TZVP basis set. ${ }^{12}$ The choice of methodology is due to its non-empirical nature, besides its good performance in the calculation of several properties in different systems. ${ }^{13-15}$

The issue of assigning formal oxidation states within the molecule has been addressed using the effective oxidation state (EOS) method of Salvador and co-workers. ${ }^{16}$ The EOS employs localized effective atomic orbitals to assign electrons to atoms, 


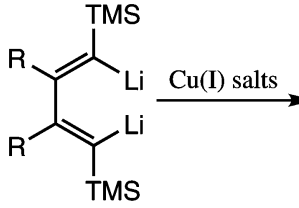

1

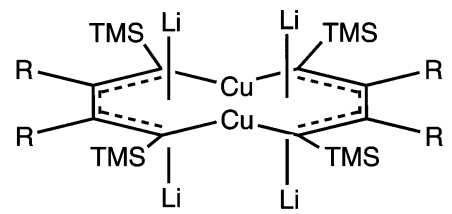

2

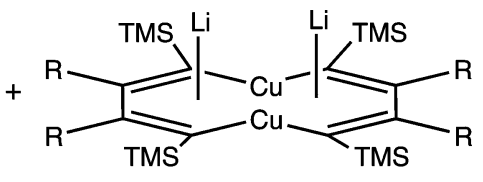

3

Fig. 1 Reaction of dilithio reagents and $\mathrm{Cu}(\mathrm{l})$ salts to produce dicupra[10]annulenes.

providing the most likely distribution of electrons as opposed to the average distribution of electrons (i.e. the atomic population). The EOS requires an atomic partition and in the present case we have used the topological fuzzy Voronoi cell (TFVC) model of the same authors. ${ }^{17}$ The TFVC consists of a partition of the electron density in atomic basins based on the fuzzy atomic Voronoi cells introduced by Becke. ${ }^{18}$ The EOS computations have been carried out using the APOST-3D software. ${ }^{19}$

The aromatic character has been assessed by means of the so-called multicenter indices, ${ }^{20}$ which have been found to perform well in describing the aromaticity of annularly delocalized electronic structures. ${ }^{21-23}$ We will focus on the normalized Giambiagi multicenter electron delocalization index $I_{\mathrm{NG}},{ }^{24}$

$$
I_{\mathrm{NG}}=\frac{\pi^{2}}{4} \frac{\left[I_{\mathrm{G}}(\mathcal{A})\right]^{1 / N}}{N N_{\pi}}
$$

where $\mathcal{A}$ is an ordered set $\left\{A_{k}\right\}_{k=1}^{N}$ of the $N$ atoms of the ring, $N_{\pi}$ stands for the number of $\pi$ electrons within the ring, and

$$
I_{\mathrm{G}}(\mathcal{A})=\int \mathrm{d} \mathbf{r}_{1} \hat{A}\left(\mathbf{r}_{1}\right) \Gamma\left(\mathbf{r}_{1} ; \mathbf{r}_{1}\right) \int \ldots \int \prod_{k=2}^{N} \hat{A}_{k}\left(\mathbf{r}_{k}\right) \Gamma\left(\mathbf{r}_{k} ; \mathbf{r}_{k+1}\right) \mathrm{d} \mathbf{r}_{k}
$$

is the non-normalized Giambiagi electron delocalization multicenter index. ${ }^{20}$ It is worth mentioning that in eqn (1), the numerical factor accounting for the straight linear correlation between $I_{\mathrm{NG}}$ and the topological resonance energy per $\pi$-electron has not been included for convenience. ${ }^{24} \Gamma\left(\mathbf{r}_{1} ; \mathbf{r}_{2}\right)$ is the spinless one-electron density matrix, and the projector operator

$$
\hat{A}_{k}\left(\mathbf{r}_{1}\right)=\int_{\Omega\left(A_{k}\right)} \delta\left(\mathbf{r}_{1}-\mathbf{r}^{\prime}\right) \mathrm{d} \mathbf{r}^{\prime}
$$

restricts the integrals in eqn (2) to the atomic basins, $\Omega\left(A_{k}\right)$, of atoms $A_{k}$, which are defined by using the quantum theory of atoms-in-molecules (QTAIM). ${ }^{25}$

This framework allows the evaluation of the number of electron-pairs shared by every pair of atoms $j$ and $k$, i.e.: the delocalization index (DI) defined as ${ }^{26}$

$$
\begin{aligned}
\delta\left(A_{j}, A_{k}\right)= & 2 \int \mathrm{d} \mathbf{r}_{1} \hat{A}_{j}\left(\mathbf{r}_{1}\right) \Gamma\left(\mathbf{r}_{1} ; \mathbf{r}_{1}\right) \times \int \mathrm{d} \mathbf{r}_{1} \hat{A}_{k}\left(\mathbf{r}_{1}\right) \Gamma\left(\mathbf{r}_{1} ; \mathbf{r}_{1}\right) \\
& -2 \int \mathrm{d} \mathbf{r}_{1} \hat{A}_{j}\left(\mathbf{r}_{1}\right) \int \mathrm{d} \mathbf{r}_{2} \hat{A}_{k}\left(\mathbf{r}_{2}\right) D\left(\mathbf{r}_{1}, \mathbf{r}_{2} ; \mathbf{r}_{1}, \mathbf{r}_{2}\right)
\end{aligned}
$$

where $D\left(\mathbf{r}_{1}, \mathbf{r}_{2} ; \mathbf{r}_{1}, \mathbf{r}_{2}\right)$ is the diagonal element of the spinless twoelectron reduced density matrix. The DIs are less sensitive to the particular atomic partition scheme chosen than atomic populations evaluated simply by integration of the electron density over the corresponding atomic basins. ${ }^{27}$ In this vein, it has also been demonstrated that multicenter indices, namely, indices between more than two atoms, should be calculated by Bader's partition. $^{28}$

Finally it is worth noting that the delocalization indices introduce a decomposition into orbital components, ${ }^{29}$ giving rise to the corresponding $\sigma$ and $\pi$ contributions to the DI and related aromaticity descriptors. ${ }^{30,31}$

The $I_{\mathrm{NG}}$ index is known to capture the extent of delocalization of the electron density, which is considered to be one salient signature of aromaticity. ${ }^{32}$ Additionally, it produces proper quantitative ordering of aromaticities of both mono- and heterocyclic compounds, ${ }^{24}$ as well as of all-metal clusters. ${ }^{33,34}$

The normalized Giambiagi multicenter electron delocalization indices $I_{\mathrm{NG}}$ were obtained from the PBE0/def2-TZVP electron density with the ESI-3D package, ${ }^{35-37}$ using the QTAIM partition. ${ }^{24}$

\section{Results}

The resulting optimum structure at the PBE0/def2-TZVP level of theory is depicted in Fig. 2. A comparison of our calculated geometrical parameters with those obtained by X-ray measurements of the dicupra[10]annulene with $\mathrm{R}=$ Phe and the TMS substituents (Table 1) reveals that all geometrical features of the ring moiety remain essentially unaltered upon replacement of the Phe and TMS substituents by $\mathrm{H}$ atoms. Thus, the bond lengths and angles of the ring are remarkably similar to those of the X-ray structure, with the largest deviations being the ones corresponding to the $\mathrm{Cu}-\mathrm{Cu}$ distance and the $\mathrm{C}-\mathrm{Cu}-\mathrm{C}$ angle, $0.21 \AA$ and 3.3 degrees, respectively. On the other hand, the $\mathrm{Cu}-\mathrm{C} 2, \mathrm{C} 2-\mathrm{C} 3$ and $\mathrm{C} 3-\mathrm{C} 4$ distances and their symmetry related ones (see the scheme in Table 1) deviate only $0.02 \AA$ with respect to the X-ray structural measurements. These results confirm that bulky substituents do not significantly modify the bonding pattern of the ring moiety, so feasible conclusions on the electronic structure of 2 can be drawn by studying the

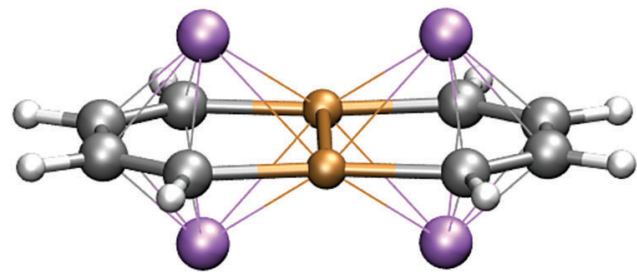

Fig. 2 Optimized geometry of $\mathbf{2}$ with $\mathrm{R}=\mathrm{H}$ and the TMS's replaced by $\mathrm{H}$ atoms. Grey: carbon, white: hydrogen, yellow: copper, magenta: lithium. 
Table 1 Geometry parameters and EOS of the optimized model dicupra[10]annulene of 2, at the PBE0/def2-TZVP level of theory. And the reported values by Wei et al. ${ }^{1}$

\begin{tabular}{lll}
\hline & 2 & Exp. \\
\hline Bond lengths (̊̊) & 2.648 & 2.438 \\
Cu-Cu & 1.899 & 1.917 \\
Cu-C2 & 2.587 & 2.145 \\
Li-Cu's & 2.979 & 1.421 \\
Li-C's & 1.391 & 1.470 \\
Li11-Li12 & 1.450 & 164.33 \\
C2-C3 & & \\
C3-C4 & 167.6 & \\
Bond angles and dihedrals (degrees) & 180.0 & \\
C10-Cu1-C2 & 0.0 & \\
C10-Cu1-Cu6-C5 & & \\
C2-C3-C4-C5 & -4 & \\
EOS & & \\
10MR & 4 & \\
Li's & 100.0 & \\
Reliability $R$ (\%) & &
\end{tabular}

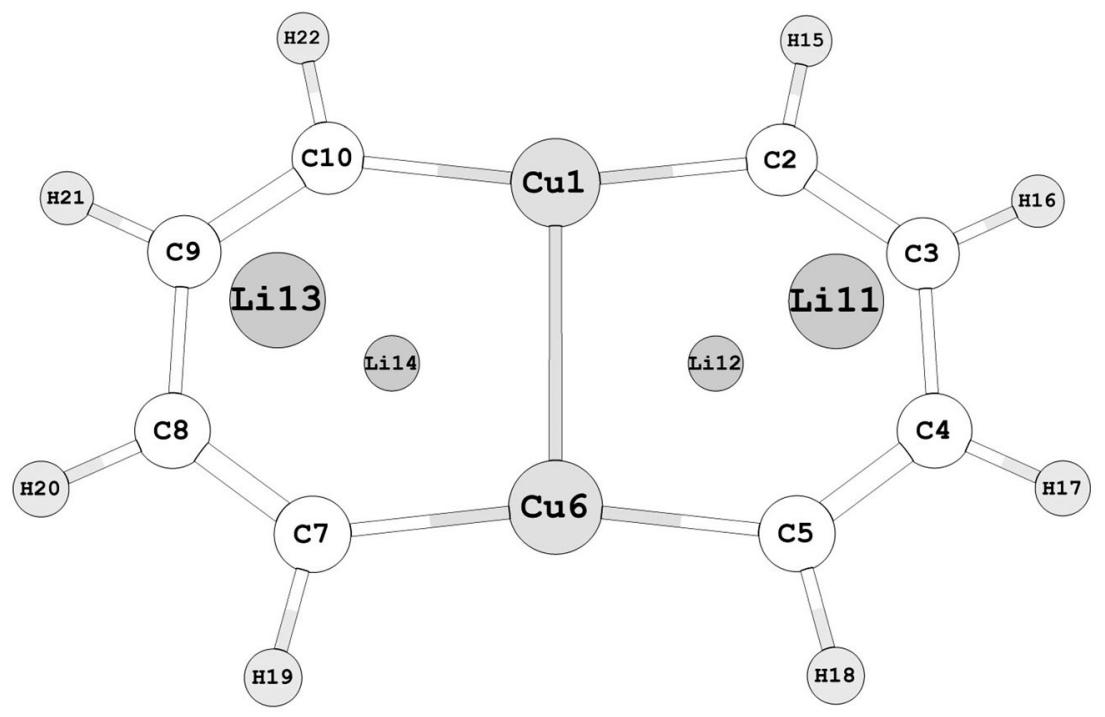

${ }^{a}$ Effective oxidation states computed considering two fragments. The former consisting of the four lithium atoms and the latter made up by the ring-like fragment.

computationally more accessible structure where such bulky substituents have been replaced by $\mathrm{H}$ atoms.

One strong point made in the original communication by Wei et al., ${ }^{1}$ is that the four lithium atoms in 2 donate one electron each to the ring-like fragment. Nonetheless, they also proved that the resulting four $\mathrm{Li}^{+}$cations play a noninnocent role in determining the geometrical structure of dicupra[10]annulenes, because they provide charge compensation to the tetraanionic dicupra[10]annulene planar moiety. Consequently, we have kept the four lithium atoms in our studies. Indeed, as Wei et al. hypothesized, the lithium atoms do donate one electron each to the ring as put forward by the EOS, shown in Table 1 . Note that the ring moiety is assigned a formal oxidation state of -4 , while each lithium bears an oxidation state of +1 . The reliability ${ }^{16}$ of such an assignment is estimated to be $100 \%$.

Furthermore, Fig. 3 shows the Bader QTAIM molecular graph of our model dicupra[10]annulene as seen from three viewpoints. Note that the bond paths, the lines connecting the nuclei bearing a bond critical point (BCP, depicted in green in Fig. 3), recover the peripheral ring molecular frame as drawn in Fig. 2, and additionally, reveal a trans-annular bond path between the copper atoms, complemented by the due cage two ring critical points (RCPs, shown as red dots in Fig. 3) corresponding to each of the two 6-membered subrings. The emerging picture is that our dicupra[10]annulene features two fused 6-membered rings, hinged at the copper atoms.

This is confirmed further by the delocalization indices (DI) computed with the aid of the ESI-3D package. ${ }^{35}$ Recall that the DIs give the number of electron pairs shared by any two atoms. It is worth noting that the numerical accuracy of the AIM calculations has been assessed using two criteria: (i) the integration of the Laplacian of the electron density within an atomic basin must be close to zero and (ii) the number of electrons in a molecule must be equal to the sum of all the electron populations of the molecule and also equal to the sum of all the localization indices and half of the delocalization 

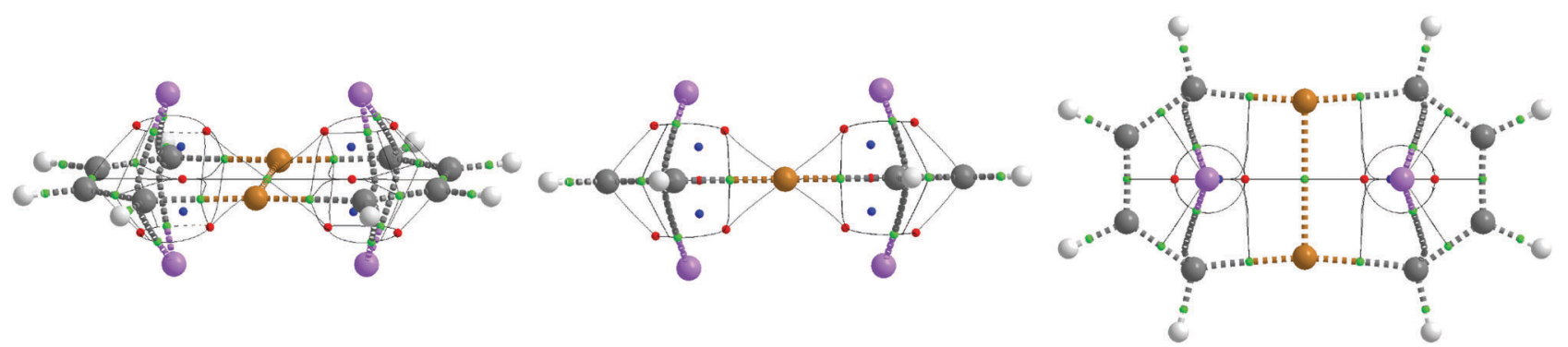

Fig. 3 Molecular graph from the Bader analysis. Bond paths are displayed in dotted lines, the BCP in green, the RCP in red, the CCP in blue and the tiny black lines connect the the RCP with the BCP.

indices in the molecule. In our case for all atomic calculations, integrated absolute values of the Laplacian were always less than 0.0012 a.u. and the errors in the calculated number of electrons were always less than 0.012 a.u.

Table 2 shows the DIs of the symmetry independent atoms of our model dicupra[10]annulene compound. Note that the Cu1-C2, Cu1-C10, Cu6-C5 and Cu6-C7 (refer to the atom numbering of the scheme shown in Table 1) atom pairs share 0.98 electron pairs, which indicate that every copper atom forms two single two-center two-electron $(2 \mathrm{c}-2 \mathrm{e})$ bonds with their neighboring carbon atoms, as the $\mathrm{H}$ atoms do with their corresponding carbon atoms, which form ordinary covalent 2c-2e $\mathrm{C}-\mathrm{H}$ bonds, DI $=0.97$ electron pairs.

The $\mathrm{C}-\mathrm{C}$ bonding shows a clear signature of a substantial degree of bond equalization, and consequently pinpoints to some degree of electron delocalization (vide infra).

The delocalization index between the copper atoms, DI = 0.46 electron pairs, is very indicative of a $\mathrm{Cu}-\mathrm{Cu}$ covalent bonding interaction.

Finally, it is worth noting that the molecular graph of our model dicupra[10]annulene shows that each $\mathrm{Li}$ atom interacts with its adjacent 6-membered ring. Thus, we have characterized two BCPs each on the two bond paths connecting each lithium with its closest two carbon atoms. This generates the two outof-plane RCPs on each side of each lithium (see Fig. 3) and the due cage critical points (CCP, shown as blue dots in Fig. 3).

Table 2 Total delocalization indices (DI) and, in parenthesis, their $\sigma$ and $\pi$ components, and the normalized Giambiagi ring currents, ING

\begin{tabular}{lll}
\hline & 2 & Naphthalene $^{a}$ \\
\hline DI (bond order) & & \\
Cu1-C2 & $0.98(0.68-0.30)$ & $1.25(0.95-0.30)$ \\
C2-C3 & $1.55(1.01-0.54)$ & $1.50(0.98-0.52)$ \\
C3-C4 & $1.24(0.94-0.30)$ & $1.29(0.96-0.33)$ \\
Cu1-Cu6 & $0.46(0.22-0.24)$ & $1.22(0.93-0.29)$ \\
C-H & $0.97(0.94-0.03)$ & $0.95(0.92-0.03)$ \\
& & \\
$I_{\text {NG }}\left(\times 10^{-3}\right)$ & 10.8 & 13.8 \\
10MR & 30.3 & 37.6 \\
6MR & & -10.9 \\
NICS $(0)$ & -14.93 & -13.8 \\
NICS $(0)_{z z}$ & -17.08 &
\end{tabular}

${ }^{a} \mathrm{Cu} 1$ and $\mathrm{Cu} 6$ stand for the transannular carbon atoms.
This is indicative of the interaction of the $\mathrm{Li}^{+}$cations with the 6-membered rings, confirming the noninnocent role played by the lithium cations.

As mentioned above, the analysis of the delocalization indices suggests some degree of electron delocalization around the ring of dicupra[10]annulene. Indeed, Wei et al. claim both, in the title and in the last section of their communication, ${ }^{1}$ that the ring moiety of the dicupra[10]annulene is aromatic. Based on the analysis of the adaptive natural density partitioning (AdNDP), formulated by Boldyrev et al. ${ }^{38}$ they conclude that the dicupra[10]annulene ring can be regarded as a Hückel aromatic $10 \pi$-electron system.

We have taken a fresh look at the aromaticity of the dicupra[10]annulene. For that purpose, we have inspected the canonical molecular orbitals and have calculated the $z$ component of their Nuclear Independent Chemical Shifts, NICS $(0)_{z z}$. Additionally, we have calculated the normalized Giambiagi ring current indices, $I_{\mathrm{NG}}$.

Our numbers tell us an extended story. They tell us that the dicupra[10]annulene ring is indeed aromatic, a $10 \pi$-electron Hückel aromatic 10-membered ring, which accounts for its perimeter aromaticity. But this perimeter ring encloses two inner rings, hinged at the $\mathrm{Cu}-\mathrm{Cu}$ bond, that are also aromatic. All in all, the aromaticity of the dicupra[10]annulene species closely resembles that of naphthalene (see below).

Fig. 4 shows the molecular orbitals of the optimized structure of our model dicupra[10]annulene. The occupied molecular orbitals of $\pi$ symmetry can readily be identified by ocular inspection, i.e.: HOMO-18, HOMO-17, HOMO-14, HOMO-11, HOMO-10, HOMO-8, HOMO-4, HOMO-2, and HOMO. Note that the HOMO-11 and the HOMO-8 do not intervene in the perimeter electron delocalization of our model dicupra[10]annulene, because they are localized on the copper atoms. Indeed, they account for the bonding and antibonding $\mathrm{Cu}-\mathrm{Cu} \pi$ interactions. According to the criteria of Schleyer et al. ${ }^{39}$ the overall Hückel aromaticity stems from the total diamagnetic contributions (see Fig. 4) from the five HOMO-18, HOMO-17, HOMO-14, HOMO-10, and HOMO, molecular orbitals. Consequently, we end up with $10 \pi$ electrons contributing to the electron delocalization along the perimeter of our dicupra[10]annulene, which fulfills the Hückel aromaticity, $4 n+2(n=2)$, electron counting rule. ${ }^{40}$ Note that the total value of the $\operatorname{NICS}(0)_{z z}(-17.08$ p.p.m.) at the centers of each ring 


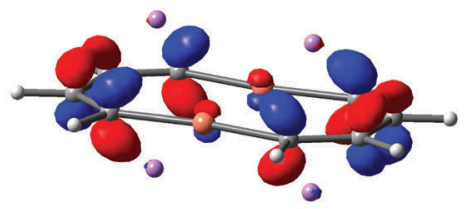

HOMO (-2.81)

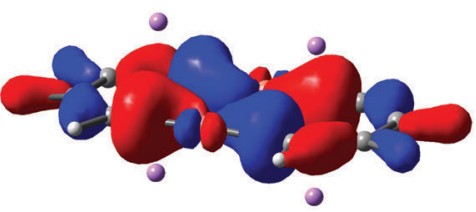

HOMO-3 (+18.59)

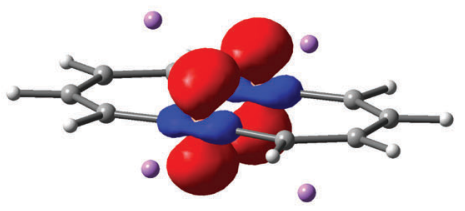

HOMO-6 (+1.38)

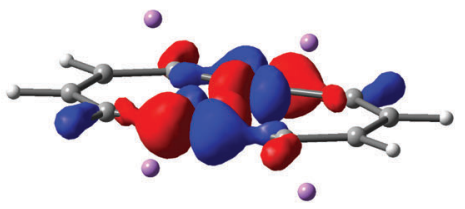

HOMO-9 (+0.02)

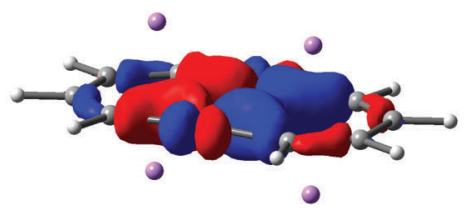

HOMO-12 (+8.77)

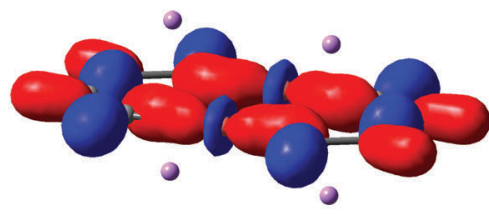

HOMO-15 (+12.99)

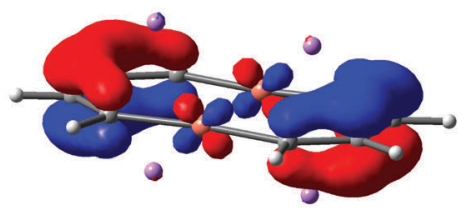

HOMO-18 (-10.75)

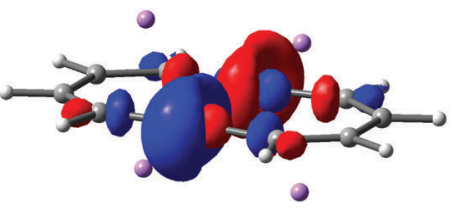

HOMO-1 (+3.19)

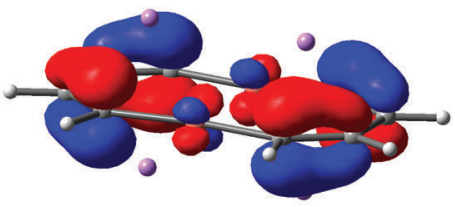

HOMO-4 (+3.01)

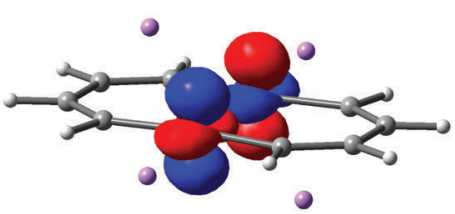

HOMO-7 (+0.31)

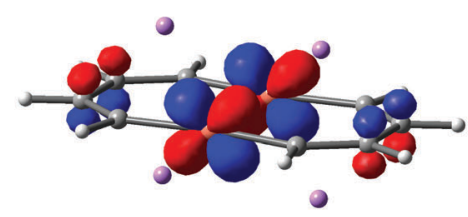

HOMO-10 (-2.46)

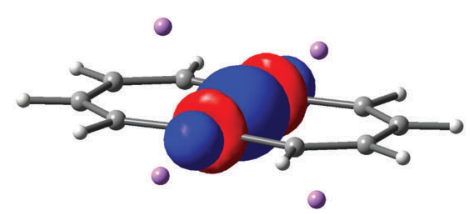

HOMO-13 (+1.79)

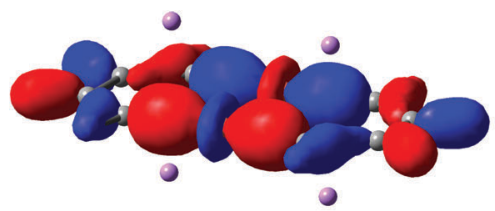

HOMO-16 (+2.11)

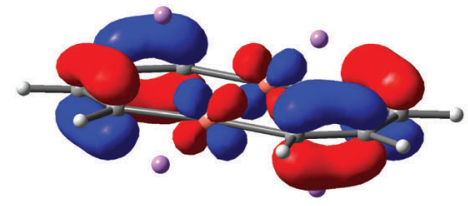

HOMO-2 (+2.40)

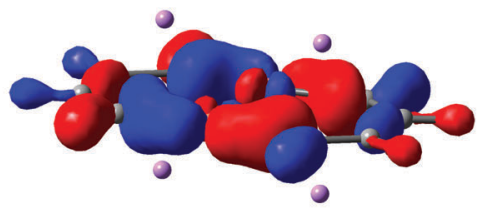

HOMO-5 (+14.67)

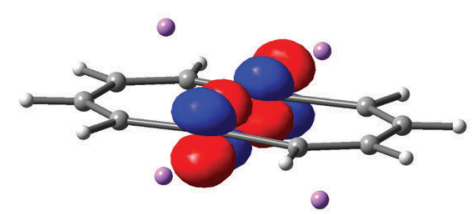

HOMO-8 (-0.96)

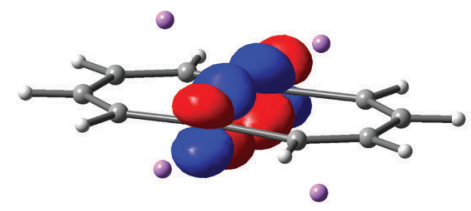

HOMO-11 (+0.41)

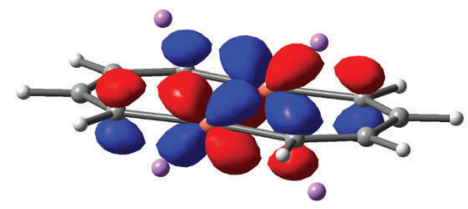

HOMO-14 (-3.28)

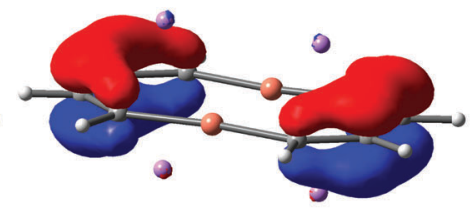

HOMO-17 (-11.83)

Fig. 4 Valence molecular orbitals of the model dicupra[10]annulene of 2. Their NICS(0) $z z$ values, in p.p.m., are given in parenthesis. Isovalue $=0.05$.

of the dicupra[10]annulene is compared with that of the naphthalene (-13.8 p.p.m.). ${ }^{36}$

However, upon close inspection of the shapes of the HOMO -4 and HOMO-2 orbitals we find it difficult not to consider both orbitals in the perimeter counting. Clearly they are $\pi$ antibonding molecular orbitals, and this is the reason for their positive $\operatorname{NICS}(0)_{z z}$ values, but they both are localized on the perimeter of the dicupra[10]annulene ring. Based on these considerations, we conclude that the perimeter aromaticity of the dicupra[10]annulene ring is substained by $14 \pi$ electrons, which also satisfies Hückel's $4 n+2$ electron counting rule. This puts forward that electron counting in metallaaromatic compounds is far from trivial, ${ }^{41}$ so it may admit more than one interpretation. 
Furthermore, the remarkable similarity of the $\pi$ delocalization indices of both species, dicupra[10]annulene and naphthalene (see the right hand numbers in the parentheses of Table 2), lends support to the claim that the inner subrings must also substain similar aromatic character, namely, the well-known $6 \pi$ electron Hückel aromaticity on the two 6-membered subrings. This picture comes along with the calculated Giambiagi normalized ring currents, $I_{\mathrm{NG}}$, for both ring-like species, dicupra[10]annulene and naphthalene, reported at the bottom of Table 2. Namely, the perimeter $I_{\mathrm{NG}}$ value of the former is 10.8, which is to be compared with the corresponding value of naphthalene $\left(I_{\mathrm{NG}}=\right.$ 13.8). Additionally, for each of the inner subrings we obtained $I_{\mathrm{NG}}=30.3$, a value that approaches the value of the naphthalene subrings $\left(I_{\mathrm{NG}}=37.6\right)$.

\section{Conclusions}

The analysis of the minimum energy geometry of our model dicupra[10]annulene, derived from 2 with the Phe and TMS substituents replaced by $\mathrm{H}$ atoms, retains the chemical bonding features of its ring moiety. Therefore, feasible conclusions on the electronic structure of 2 can be drawn by focusing on the computationally more accessible model compound.

The analysis of the effective oxidation states of the lithium atoms and the ring moiety fragments is consistent with a full transfer of one electron from each of the four lithium atoms to the ring. Additional inspection of the molecular graph obtained from Bader's QTAIM analysis of the calculated PBE0/def2-TZVP electron density reveals the interactions of the resulting four lithium cations with the ring-like moiety, putting forward the noninnocent role played by the four $\mathrm{Li}^{+}$atoms in determining the structure of the title compound.

Furthermore, these results complemented with the analysis of the delocalization indices are very supportive of a substantial degree of bond equalization between the carbon atoms of the ring, and of a covalent $\mathrm{Cu}-\mathrm{Cu}$ interaction, yielding a structure which is best seen as a 10-membered ring enclosing two 6-membered rings hinged at the $\mathrm{Cu}-\mathrm{Cu}$ bond.

Inspection of the valence molecular orbitals shows that the perimeter aromaticity of our model dicupra[10]annulene is substained by seven such molecular orbitals, giving rise to a $14 \pi$-electron Hückel aromatic perimeter ring. However, two of them have positive $\operatorname{NICS}(0)_{z z}$ values, suggesting that the overall perimeter aromaticity of dicupra[10]annulene can be attributed to the diamagnetic contributions of only five molecular orbitals. This gives rise to a $10 \pi$-electron Hückel aromatic perimeter ring, which compares with that of the naphthalene as reflected by the similarity of the calculated Giambiagi normalized perimeter ring currents. Further analysis of the $\pi$ components of the delocalization indices puts forward the $6 \pi$-electron aromaticity of the two inner 6-membered rings, based on the close similarity of these values to those of the naphthalene. The latter is also consistent with the calculated values of the Giambiagi normalized ring current indices of these inner rings of both our model dicupra[10]annulene and naphthalene.
Consequently, we have found that the perimeter aromaticity of the dicupra[10]annulene ring is consistent with both 10 and $14 \pi$-electron Hückel aromatic 10-membered rings which enclose two $6 \pi$-electron aromatic inner rings, hinged at the $\mathrm{Cu}-\mathrm{Cu}$ bond. The aromaticity of the dicupra[10]annulene closely resembles that of the naphthalene. This work puts forward that the salient aromatic character of dicupra[10]annulene does not correspond to an aromatic analogue of [10]annulene with only one peripheral ring current, but to a metalated naphthalene with three aromatic ring currents. Therefore, the compound can be best seen as a metalla-polyacene, a potential building block for larger structures such as metalated nanotubes.

\section{Acknowledgements}

The authors are thankful for the technical and human support provided by IZO-SGI SGIker of UPV/EHU and European funding (ERDF and ESF). R. G.-A. gratefully acknowledges Consejo Nacional de Ciencia y Tecnología (CONACYT) for the postdoctoral fellowship. Financial support comes from Eusko Jaurlaritza (Basque Government) through the project IT588-13, and from the Spanish Office of Scientific Research through Projects No. CTQ2012-38496-C05-01, CTQ2014-52525-P and CTQ2015-67660-P.

\section{References}

1 J. Wei, Y. Zhang, Y. Chi, L. Liu, W.-X. Zhang and Z. Xi, J. Am. Chem. Soc., 2016, 138(1), 60-63.

2 J. M. Mercero, I. Infante and J. M. Ugalde, in Aromaticity and Metal Clusters, ed. P. K. Chattaraj, CRC Press, Boca Raton, FL, USA, 2011, ch. 16.

3 F. Feixas, E. Matito, J. Poater and M. Solà, Wiley Interdiscip. Rev.: Comput. Mol. Sci., 2013, 3, 105-122.

4 J. M. Mercero, A. I. Boldyrev, G. Merino and J. M. Ugalde, Chem. Soc. Rev., 2015, 44(18), 6519-6534.

5 I. Fernández, G. Frenking and G. Merino, Chem. Soc. Rev., 2015, 44(18), 6452-6463.

6 M. Mauksch and S. B. Tsogoeva, Chem. - Eur. J., 2010, 16(26), 7843-7851.

7 A. F. Dalebrook and L. J. Wright, Adv. Organomet. Chem., 2012, 60, 93-177.

8 D. L. Thorn and R. Hoffmann, Nouv. J. Chim., 1979, 3(1), 39-45.

9 Q. Zhuo, X. Zhou, H. Kang, Z. Chen, Y. Yang, F. Han, H. Zhang and H. Xia, Organometallics, 2016, 1497-1504.

10 M. J. Frisch, G. W. Trucks, H. B. Schlegel, G. E. Scuseria, M. A. Robb, J. R. Cheeseman, J. A. Montgomery, Jr., T. Vreven, K. N. Kudin, J. C. Burant, J. M. Millam, S. S. Iyengar, J. Tomasi, V. Barone, B. Mennucci, M. Cossi, G. Scalmani, N. Rega, G. A. Petersson, H. Nakatsuji, M. Hada, M. Ehara, K. Toyota, R. Fukuda, J. Hasegawa, M. Ishida, T. Nakajima, Y. Honda, O. Kitao, H. Nakai, M. Klene, X. Li, J. E. Knox, H. P. Hratchian, J. B. Cross, V. Bakken, C. Adamo, J. Jaramillo, R. Gomperts, R. E. Stratmann, O. Yazyev, A. J. Austin, R. Cammi, 
C. Pomelli, J. W. Ochterski, P. Y. Ayala, K. Morokuma, G. A. Voth, P. Salvador, J. J. Dannenberg, V. G. Zakrzewski, S. Dapprich, A. D. Daniels, M. C. Strain, O. Farkas, D. K. Malick, A. D. Rabuck, K. Raghavachari, J. B. Foresman, J. V. Ortiz, Q. Cui, A. G. Baboul, S. Clifford, J. Cioslowski, B. B. Stefanov, G. Liu, A. Liashenko, P. Piskorz, I. Komaromi, R. L. Martin, D. J. Fox, T. Keith, M. A. Al-Laham, C. Y. Peng, A. Nanayakkara, M. Challacombe, P. M. W. Gill, B. Johnson, W. Chen, M. W. Wong, C. Gonzalez and J. A. Pople, Gaussian 09, Gaussian Inc., Wallingford, CT, 2009.

11 C. Adamo and V. Barone, J. Chem. Phys., 1999, 110(13), 6158-6170.

12 F. Weigend and R. Ahlrichs, Phys. Chem. Chem. Phys., 2005, 7(18), 3297-3305.

13 M. Steinmetz and S. Grimme, ChemistryOpen, 2013, 2(3), 115-124.

14 S. S. Leang, F. Zahariev and M. S. Gordon, J. Chem. Phys., 2012, 136, 104101.

15 J. O. C. Jimenez-Halla, E. Matito, M. Sola, H. Braunschweig, C. Horl, I. Krummenacher and J. Wahler, Dalton Trans., 2015, 44, 6740-6747.

16 E. Ramos-Cordoba, V. Postils and P. Salvador, J. Chem. Theory Comput., 2015, 11(4), 1501-1508.

17 P. Salvador and E. Ramos-Cordoba, J. Chem. Phys., 2013, 139, 071103.

18 A. D. Becke, J. Chem. Phys., 1988, 88(4), 2547-2553.

19 P. Salvador and E. Ramos-Cordoba, Program APOST-3D, Version 2.00, Girona, 2014.

20 M. Giambiagi, M. S. de Giambiagi, C. D. dos Santos Silva and A. P. de Figuereido, Phys. Chem. Chem. Phys., 2000, 2, 3381-3392.

21 F. Feixas, E. Matito, J. Poater and M. Solà, Chem. Soc. Rev., 2015, 44, 6389-6646.

22 E. Matito, Phys. Chem. Chem. Phys., 2016, 18(17), 11839-11846.

23 J. M. Mercero, E. Matito, F. Ruipérez, I. Infante, X. Lopez and J. M. Ugalde, Chem. - Eur. J., 2015, 21(27), 9610-9614.
24 J. Cioslowski, E. Matito and M. Solà, J. Phys. Chem. A, 2007, 111, 6521-6525.

25 R. F. W. Bader and P. M. Beddall, J. Chem. Phys., 1972, 56, 3320-3329.

26 R. F. W. Bader and M. E. Stephens, Chem. Phys. Lett., 1974, 26, 445 .

27 E. Matito, J. Poater, M. Solà, M. Duran and P. Salvador, J. Phys. Chem. A, 2005, 109, 9904-9910.

28 W. Heyndrickx, P. Salvador, P. Bultinck, M. Solà and E. Matito, J. Comput. Chem., 2011, 32, 386-395.

29 E. Matito, M. Solà, M. Duran and P. Salvador, J. Phys. Chem. A, 2006, 110, 5108-5113.

30 F. Feixas, E. Matito, M. Solà and J. Poater, Phys. Chem. Chem. Phys., 2010, 12(26), 7126-7137.

31 M. Güell, E. Matito, J. M. Luis, J. Poater and M. Solà, J. Phys. Chem. A, 2006, 110(40), 11569-11574.

32 P. Bultinck, M. Rafat, R. Ponec, B. V. Gheluwe, R. Carbo-Dorca and P. Popelier, J. Phys. Chem. A, 2006, 110, 7642-7648.

33 F. Feixas, E. Matito, M. Duran, J. Poater and M. Solà, Theor. Chem. Acc., 2011, 128, 419-431.

34 F. Feixas, J. Jiménez-Halla, E. Matito, J. Poater and M. Solà, J. Chem. Theory Comput., 2010, 6, 1118-1130.

35 E. Matito, ESI-3D: Electron sharing indices program for 3D molecular space partitioning, 2015. Available in http:// ematito.webs.com/programs.htm+.

36 E. Matito, M. Duran and M. Solà, J. Chem. Phys., 2005, 122, 014109.

37 E. Matito, M. Solà, P. Salvador and M. Duran, Faraday Discuss., 2007, 135, 325-345.

38 D. Y. Zubarev and A. I. Boldyrev, Phys. Chem. Chem. Phys., 2008, 10(34), 5207-5217.

39 C. Zhu, M. Luo, Q. Zhu, J. Zhu, P. v. R. Schleyer, J. I.-C. Wu, X. Lu and H. Xia, Nat. Commun., 2014, 5, 3265.

40 E. Hückel, Z. Phys., 1931, 70(3-4), 204-286.

41 I. Fernández and G. Frenking, Chem. - Eur. J., 2007, 13, 5873-5884. 